\title{
The Cytotoxic and Anti-Migratory Properties of Caesalpinia sappan and Ficus septica, in Combination with Doxorubicin on 4T1 TNBC Cells with Nephroprotective Potential
}

\author{
Sari Haryanti', Ummi Maryam Zulfin², Irfani Aura Salsabila², Febri Wulandari², \\ Edy Meiyanto $2,3 *$
}

\begin{abstract}
Objective: To evaluate the anti-cancer properties of Caesalpinia sappan and Ficus septica in combination with doxorubicin on 4T1 cells, confirm their nephroprotective activities, and predict the molecular targets of the underlying mechanisms. Methods: The cytotoxic activities of all extracts and doxorubicin were determined by MTT assay followed by cell cycle and apoptosis analysis using flow cytometry. Immunoblotting was used to determine the protein expressions. The proteins involved in the cell proliferation and migration were analyzed through bioinformatics approaches, whereas, the interaction between compounds and protein targets was observed through molecular docking. Furthermore, the effect of the extracts on cell migration was analyzed by scratch wound healing assay. The intracellular ROS after treatment with extracts was observed using DCFDA staining flow cytometry. Results: Both ECS and EFS performed cytotoxic properties and significantly enhanced doxorubicin's cytotoxic effects against 4T1 cells. However, these cytotoxic activities did not correlate with the cell cycle progression. On the contrary, the combination treatment caused apoptosis that may correlate with the decreasing of I $\mathrm{B}$ a phosphorylation, indicating that all agents targeted the inhibition of NF- $\mathrm{KB}$ activation. The combination treatments also inhibited cell migration and decreased MMP-9 expression. TNBC proliferation and metastasis needed at least 54 proteins to be activated, some of them are related to NF- $\kappa \mathrm{B}$ activation. The inhibitory effect of ECS correlated with the interaction of brazilin and brazilein to IKK, a kinase protein that plays a role in IKBa phosphorylation. In addition, ECS and EFS reduced ROS expression in Vero cells caused by doxorubicin. Conclusion: In conclusion, ECS and EFS effectively enhanced the cytotoxic effect of doxorubicin and inhibit cell migration on 4T1 cells and these activities may correlate to the inhibitory effect of $\mathrm{NF}-\kappa \mathrm{B}$ activation. ECS and EFS also exhibit ROS suppressing effect on Vero cells that may be beneficent to reduce nephrotoxicity of chemotherapeutic treatment.
\end{abstract}

Keywords: Caesalpinia sappan-Ficus septica- cancer growth suppression-Cytotoxic combination (co-treatment)

Asian Pac J Cancer Prev, 23 (2), 743-753

\section{Introduction}

The triple negative breast cancer (TNBC) subtype of breast cancer is a kind of aggressive breast cancer with limited treatment choices (Yin et al., 2020). This strain exhibits relatively higher resistance to standard chemotherapy, particularly anthracyclines and taxanes, especially in the metastatic stage (Wahba and El-Hadaad, 2015). Standard chemotherapy has a shorter response duration, a higher recurrence rate, and shorter patient survival (André and Zielinski, 2012). Existing chemotherapeutic agents have a number of drawbacks, including resistance, side effects, and insufficient efficacy in advanced cancers. Doxorubicin is one of these groups of drugs. Moreover, in metastatic breast cancer types, doxorubicin induces lamellipodia formation which plays an important role in cell migration (Meiyanto et al., 2019). Figuring out the best regiment and use of cochemotherapeutic agents to suppress or even alleviate the side effects of chemotherapy is an interesting challenge.

Targeting the cell cycle to inhibit proliferation as well as promoting apoptosis is one of the important ways to disturb cancer development (Haryanti et al., 2016). On the other hand, targeting cell migration through inhibition of its regulatory proteins also gives significance in inhibiting cell invasion and metastasis (Landis-Piwowar

${ }^{1}$ Medicinal Plant and Traditional Medicine Research and Development Center, Tawangmangu, Central Java, Indonesia. ${ }^{2}$ Cancer Chemoprevention Research Center, Faculty of Pharmacy, Universitas Gadjah Mada (UGM), Yogyakarta, Indonesia. ${ }^{3}$ Laboratory of Macromolecular Engineering, Department of Pharmaceutical Chemistry, Faculty of Pharmacy, UGM, Yogyakarta, Indonesia. *For Correspondence: edy_meiyanto@ugm.ac.id 
and Iyer, 2014). Proliferation and metastasis are important events in carcinogenesis that involve many regulators, including several transcription factors. Namely, NFкB is a transcription factor that actively participates in both stages due to its pivotal role in directly activating several genes involved in cell division and metastasis (Wang et al., 2015). Inhibition of NFאB activation is expected to downregulate proteins required in proliferation and metastasis such as Cyclin D1 which plays a role in proliferation, an anti-apoptotic protein $\mathrm{BCl} 2$, and MMPs that are indispensable proteins in metastasis (Qin et al., 2012; Zhao et al., 2017). Doxorubicin as a

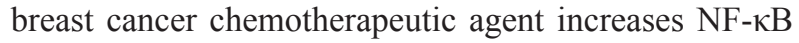
activation through the formation of free radicals or Reactive Oxygen Species (ROS) that can damage cells non-selectively (Finn and Kemp, 2012). This effect is one of the causes of nephrotoxicity and cardiotoxicity, one of which is mediated by the Rac1 protein. This is also one other reason, presumably, that doxorubicin induces cell migration due to the increasing Racl expression (Ma et al., 2013). This pleiotropic effect of doxorubicin remains the challenge to find out a co-treatment against doxorubicin, especially to target cancer cells' proliferation and migration and reducing the nephrotoxicity.

One approach to finding chemopreventive compounds is through the exploration of natural sources (Cragg and Pezzuto, 2016). Several plants with specific molecular targets can be combined to optimize cancer therapy, especially for metastatic cancer. The combination of plants with different targets of action is expected to be a reliable chemopreventive and co-chemotherapeutics agent (Rizeq et al., 2020). Some plants that are easy to grow and are found in various parts of Indonesia contain compounds that are potential as chemopreventive agents. These include Sappan woods (Caesalpinia sappan L.) and awar-awar (Ficus) leaves (Ficus septica Burm. F) (Hidayati et al., 2018; Jenie et al., 2018).

The main chemicals in Sappan wood extract, brazilein and brazilin, have been shown to have potent cytotoxic effects on HER2 expressing breast cancer cells in vitro by inhibiting NFKB activation and lowering MMP2 expression (Handayani et al., 2017; Hsieh et al., 2013; Jenie et al., 2018). These activities are mediated by cell cycle inhibition and apoptosis induction through different signal transduction (Handayani et al., 2017; Jenie et al., 2018). Awar-awar (Ficus) leaves exhibit an immunomodulatory effect by reducing lymphocyte density, phagocytic activity, and macrophage capacity, as well as increasing T cell CD8+ levels in the blood and decreasing IL-10 expression in the spleen (Nugroho et al., 2012). Moreover, a combination of awar-awar extract which contains tylophorine, and black cumin which contains thymoquinone synergistically inhibited the growth of HeLa cervical cancer cell line (Santoso et al., 2019). The differences in the mechanism of action of these various plants supposed to have the potential to increase the effectiveness of doxorubicin against TNBC and lowering the risk of its nephrotoxicity.

In this study, we examined the cytotoxic and anti-migratory activities of Sappan woods and Ficus leaves on 4T1, a model of TNBC cells. The potential of this combination of plants is explored to increase the activity of the chemotherapeutic agent doxorubicin, either through the induction of apoptosis, inhibition of the cell cycle, or inhibition of migration in the 4T1 breast cancer cell line. Furthermore, the mechanism of antiproliferative and antimetastatic action of the combination of plants with doxorubicin was investigated through bioinformatics and biochemical approaches. Whereas, the Vero cell line was used as a normal kidney cell to evaluate the inhibitory potential of the herbs on doxorubicin-induced ROS generation in kidney cells. Expectantly, all the data would comprehensively figure out the potential activity of those herbs, in single or in combination, as co-chemotherapeutic agents for TNBC with cytoprotective potential on kidney cellular damage, in vitro, in silico, and their molecular mechanisms.

\section{Materials and Methods}

\section{Materials and extraction}

The powder of Sappan wood (Caesalpinia sappan L.) and awar (Ficus septica Burm. F.) leaves were prepared from Medicinal Plant and Traditional Medicine Research and Development Center (MPTMRDC), Tawangmangu, Central Java, Indonesia. The powder of both simplisia were macerated in $96 \%$ ethanol and dried at $40^{\circ} \mathrm{C}$ to obtain dried extracts of sappan wood (ECS) and ficus leaves (EFS). In DMSO (Sigma) as the stock solution, both extracts and doxorubicin (Sigma) were dissolved. The working solution was prepared through the dilution of the stock solution with culture medium to achieve the respective concentrations.

\section{Cell lines and culturing}

The 4T1 breast cancer and Vero normal kidney cell lines as collected by Cancer Chemoprevention Research Center (CCRC), Universitas Gadjah Mada (UGM) were maintained using Gibco's Dulbecco Minimum Essential Medium (DMEM) containing 10\% fetal bovine serum/ FBS (Gibco), 1\% penicillin-streptomycin (Gibco). All of the cells were incubated at $37^{\circ} \mathrm{C}$ in a $5 \% \mathrm{CO}_{2}$ incubator.

\section{Cytotoxic assay}

Cytotoxic evaluation was conducted based on the metabolically active cells to convert 3-(4,5-dimethylthiazol2-yl)-2,5-diphenyltetrazolium bromide (MTT) into an oxidative form of formazan that can be detected using visible light. A series of the respective concentrations of extracts or doxorubicin were used to treat the respected cell lines (4T1 or Vero) that were seeded in a 96-well plates at the amount of $1 \times 10^{4}$ cells per well and then were incubated for $24 \mathrm{~h}$. The same setting was used for either alone or combination treatments. After incubation, discharging the medium, and washing with PBS (Sigma), each well received $0.5 \mathrm{mg} / \mathrm{mL}$ MTT (Sigma) in the medium and were incubated for 3-4 h, followed by adding $10 \%$ SDS in $0.01 \mathrm{~N} \mathrm{HCl}$ and incubated overnight without light. At $595 \mathrm{~nm}$, the absorbance was measured with a microplate reader (Biorad). The absorbances were converted into cell viabilities by comparing between the treatment and untreated cells to obtain the cytotoxic profiles among the 
treatments.

Cell cycle modulation and apoptosis induction by flow cytometry assay

In a 6 -well plate, $5 \times 10^{5} 4 \mathrm{~T} 1$ cells were cultured and incubated for $24 \mathrm{~h}$ and subjected to be treated with the extracts or doxorubicin, either single or in combination. After $24 \mathrm{~h}$ incubation, cells were collected through detaching with trypsin-EDTA, and washing with phosphate-buffered saline (PBS) prior to centrifugation for 5 minutes at $500 \mathrm{rpm}$. The obtained cells were then fixed in $70 \%$ cold ethanol for 30 minutes for cell cycle analysis. Afterwards, the cells were collected through centrifugation for $5 \mathrm{~min}$ at $500 \mathrm{rpm}$ prior to DNA staining with propidium iodide (Sigma), $20 \mathrm{ng} / \mathrm{mL}$ RNAse (Roche), and $0.1 \%$ TritonX 114 (Sigma) for $15 \mathrm{~min}$ in the dark tube prior to analysis on a BD Accuri C6 flow cytometer. The apoptosis measurement was performed using Annexin V FITC and Propidium Iodide (BD Pharmingen) treatment in the dark for 15 min before being examined using a BD Accuri C6 Flow cytometer.

\section{Western Blot}

In a $10 \mathrm{~cm}$ tissue culture dish, $10^{6} 4 \mathrm{~T} 1$ cells were grown and incubated for $24 \mathrm{~h}$ prior to the extracts or doxorubicin treatments, either separately or in combination treatments. Protein was isolated using Proprep (Intron Biotechnology) and separated using SDS-PAGE in a $14 \%$ acrylamide gel. The membrane was added with either a rabbit monoclonal antibody to PARP1 (Cell Signaling D64E10) or a mouse monoclonal antibody to $\beta$-actin (Cell Signaling D64E10) (Santa Cruz sc47778) and incubated overnight at $4^{\circ} \mathrm{C}$ after transfer to a PVDF membrane. After 1 hour of incubation with secondary antibodies anti-rabbit (Santa Cruz sc2357) and anti-mouse (Santa Cruz sc516102), the protein bands were detected using Enhanced Chemiluminescence (ECL, Amersham) and identified by Luminograph. The relative protein levels were computed using the amount of actin protein as a reference.

\section{Scratch assay}

In 6 -well plate, approximately $7 \times 10^{4}$ cells/well were grown and incubated for $24 \mathrm{~h}$. The cells were then scraped with a sterile $200 \mu \mathrm{L}$ tip. The scratched cells were rinsed in PBS before treatment with the extracts or doxorubicin, either separately or in combination, in 10 $\%$ FBS supplemented-culture medium. At $0,18,24$, and $42 \mathrm{~h}$, the cell migration was examined under an inverted microscope and photographed with a digital camera. Image J software was used to evaluate the scratch area and wound closure at various time intervals.

\section{Gelatin zymography}

In a $3 \mathrm{~cm}$ tissue culture dish, $3 \times 1054 \mathrm{~T} 1$ cells were seeded and incubated for $24 \mathrm{~h}$. For 24 hours, the cells were given treatments of the extracts and doxorubicin, either separately or in combination. The culture medium was transferred to a microtube and centrifuged at $4{ }^{\circ} \mathrm{C}$ at $10,000 \mathrm{rpm}$ for two min. SDS-PAGE containing $0.1 \%$ gelatin copolymer was used to separate the supernatant in a microtube. After that, the gel was dyed with Coomassie blue. On a dark blue background, the areas of enzyme activity appeared transparent. ImageJ software was used to examine the band area.

\section{Bioinformatics study} Analysis of gene markers

The associated genes involved in TNBC with the keywords proliferation and metastasis were examined using Genecards (https://www.genecards.org/), an open public database of gene expression profiling. The filtered genes are considered as gene markers in TNBC. All statistical information was taken directly from the database.

\section{Analysis of protein targets for brazilin, brazilein, and tylophorine}

Swis s TargetPrediction (http://ww . swisstargetprediction.ch/) database was employed to determine which proteins are most likely to be targets of brazilin, brazilein, and tylophorine. The canonical structures of the compounds were gathered from the Pubchem database (https://pubchem.ncbi.nlm.nih.gov/). Using Interactivenn (http://www.interactivenn.net/), the protein targets were then analyzed by the cross-section to the gene markers in TNBC. The intercept proteins were selected for the most related to its proliferative and metastatic markers then examined further using molecular docking.

\section{Molecular docking}

Molecular Operating Environment (MOE) version 2010.10 software (licensed for Faculty of Pharmacy Universitas Gadjah Mada) was used to analyze the binding interaction between brazilin, brazilein, and tylophorine toward MMP9, MMP2, PTGS2, and IKK. The validation of their molecular binding was calculated based on the Root Mean Square Deviation (RMSD), and the binding characteristic was visualized in protein-ligand interaction. The PDB ID of MMP-9, MMP-2, PTGS2, and IKK were $2 \mathrm{OW} 1,1 \mathrm{HOV}, 4 \mathrm{M} 11$, and $4 \mathrm{KIK}$ respectively. The score of the placements were set up based on the triangle matcher and London $\Delta \mathrm{G}$ with the refining of the docking findings from ten different retain settings through the force field approach. Chemical builders in MOE were used to visualize the structure of brazilin, brazilein, and tylophorine. Afterwards, the structures are prepared for structural energy minimization and conformational structure generation in MOE. The molecular docking study was carried out on each protein's native ligand binding site. Each drug's docking score and binding visualization to the target proteins were used to explain the affinity reflected by the docking score.

\section{Data analysis}

One-way ANOVA followed by a Bonferroni post-hoc test with SPSS v.16 was used to analyze all data with triplicate measurements. The data are presented as the mean SD of three independent experiments. $\mathrm{P}<0.05$ was used to determine statistically significant differences. 


\section{Results}

Single Cytotoxic effects of Caesalpinia sappan Wood Extract (ECS), Ficus septica Extract (EFS), and Dox on $4 T 1$ cells

We performed an MTT assay to evaluate the cytotoxicity of ECS, EFS, and doxorubicin employing 4T1, a TNBC and elicit metastatic characteristics. Both extracts, ECS and EFS, give cytotoxic activities against $4 \mathrm{~T} 1$ cells with the $\mathrm{IC}_{50}$ of $9.3 \mu \mathrm{g} / \mathrm{mL}$ and $1.3 \mu \mathrm{g} / \mathrm{ml}$ respectively (Figure 1a). EFS exhibited a more effective suppress of 4T1 cells growth. Whereas, doxorubicin was more effective against $4 \mathrm{~T} 1$ cells with the $\mathrm{IC}_{50}$ value of $1.2 \mu \mathrm{g} / \mathrm{mL}$ (Figure 1a). The cytotoxic potency of both extracts is classified as strong cytotoxic when the $\mathrm{IC}_{50}$ values are less than $20 \mu \mathrm{g} / \mathrm{mL}$. Therefore, both extracts are promising to explore further for their molecular and physiological mechanisms.

The purpose of this investigation is to evaluate the cytotoxic effects of their co-treatment on 4T1 cells. We used doses under the $\mathrm{IC}_{50}$ of each agent to be combined, in double and triple combination, and subjected to treat the cells. The cytotoxic potency of their combinations was evaluated under the significant differences of the percentage cell viability between the single treatments and the combination treatments. The results showed that all the single treatments did not change the cell viabilities compared to untreated cells. However, all of the combination treatments decreased the cell viability significantly $(p<0.001)$, meaning that both extracts enhanced the cytotoxic effect of doxorubicin significantly in the lower dose of the $\mathrm{IC}_{50}$ value (Figure 1b). These results indicated that ECS and EFS were able to increase the cytotoxic effect of doxorubicin against 4T1 cells.

Effect of ECS, EFS, Dox, as well as their Cell cycle progression is the ma combinations on cell cycle progression

Effect of ECS, EFS, Dox, as well as their Cell cycle progression is the main event in cell proliferation that is targeted by many cytotoxic agents. Since ECS and EFS performed a relatively strong cytotoxic effect on 4T1 cells and enhanced the cytotoxic effect of Dox, we then evaluated their effects on cell cycle progression using flow cytometry analysis in single and in combination, including with doxorubicin. We found that there was no significant differences among all treatments, except the combination treatment of ECS and EFS markedly in the S phase (Figure 2). These results showed that the combination of ECS and EFS, including with Dox, did not affect the cell cycle profiles that may irrespective of the cell death effects.

\section{Effect of ECS, EFS, Dox, as well as their combinations on apoptosis induction}

Since their combination did not affect the cell cycle progression, we proposed that they might affect apoptosis or cell death directly. However, flow cytometry analysis results showed that only doxorubicin and its combination induced cell death in some evidence such as early apoptosis, late apoptosis, and necrosis. We found that the combination of ECS and EFS did not increase cell death. However, the three combinations increased cell death through apoptosis or necrosis that might correlate to their cytotoxic effects (Figure 3 ).

\section{Evaluation of PARP-1 and I $\mathrm{B} \alpha$ expression}

We then evaluated the protein expression as the essential markers related to apoptosis and cell proliferation. We chose PARP-1 protein that is usually cleaved by caspase-3 as the main indicator that cells undergo apoptosis, whereas the phosphorylated $\mathrm{I} \kappa \mathrm{B} \alpha$ is the indicator for $\mathrm{NF}-\kappa \mathrm{B}$ activation that commonly occurs in active cell proliferation. We found that there was no PARP-1 cleaved except for a slight appearance in doxorubicin treatment (Figure 4). This evidence might correlate to the effect of both extracts not inducing apoptosis of 4T1 cells significantly. However, all treatments decreased the

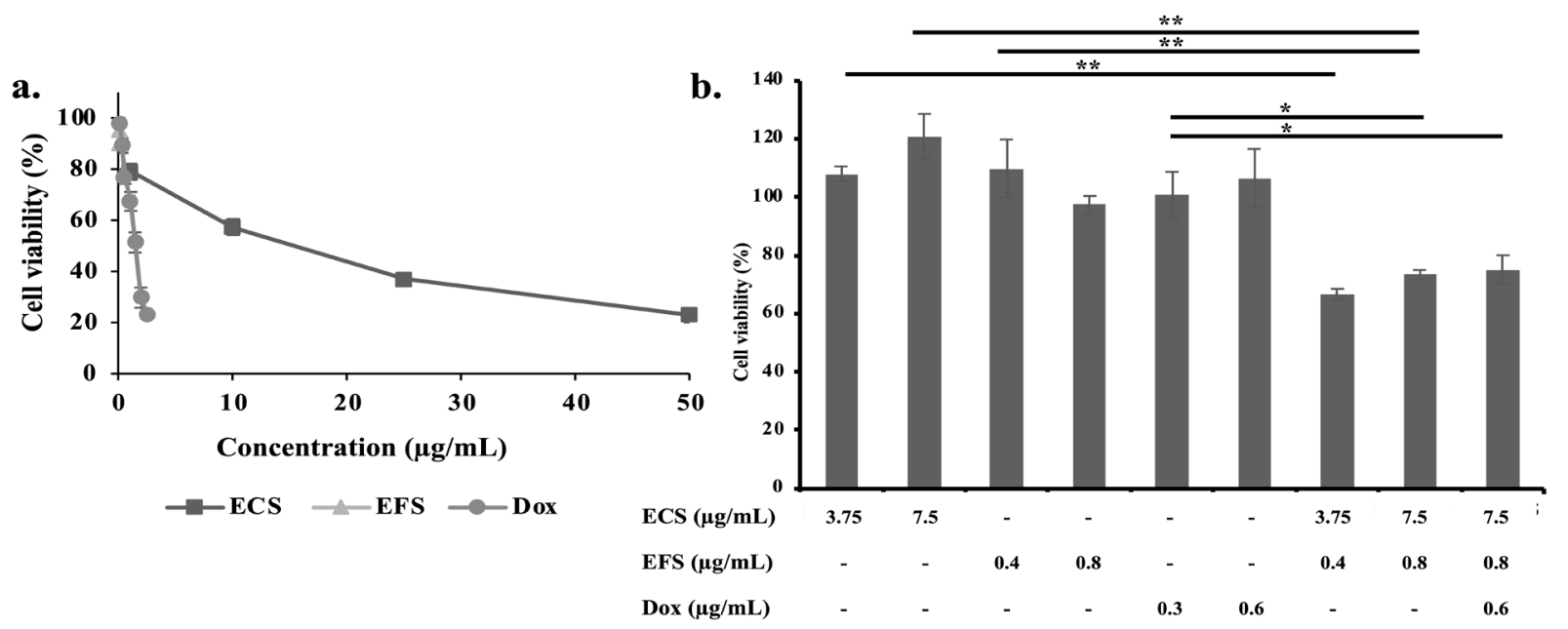

Figure 1. Cytotoxic Effects of Caessalpinnia Sappan Wood Extract (ECS), Ficus Septica Extract (EFS), and Doxorubicin (Dox) on 4T1 Cells. MTT assay was used to measure the cytotoxic effect of all the treatments for $24 \mathrm{~h}$ as described in the method. (a) Growth inhibitory profiles of ECS, EFS, and Dox treatments on $4 \mathrm{~T} 1$ cells. The IC values $_{50}$ of ECS, EFS, and Dox were $9.3 \mu \mathrm{g} / \mathrm{mL}, 1.3 \mu \mathrm{g} / \mathrm{mL}$, and $1.2 \mu \mathrm{g} / \mathrm{mL}$, respectively. (b.) Growth inhibitory profiles of $4 \mathrm{~T} 1$ cells after combination treatment of ECS, EFS, and Dox. $(* \mathrm{p}<0.01, * * \mathrm{p}<0.001)$. 


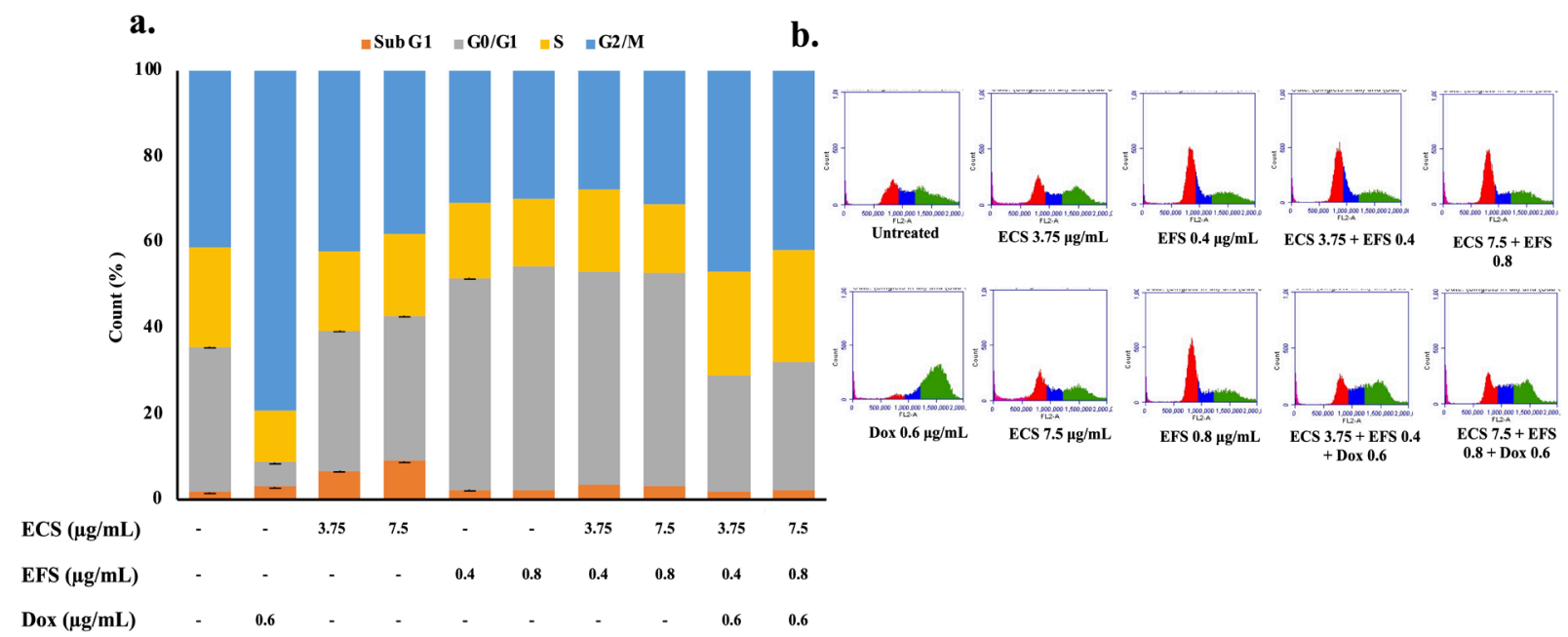

Figure 2. Cell Cycle Distribution Effects of ECS, EFS, Dox, as well as Their Combinations Treatment on 4T1 Cells. The assay was conducted based on flow cytometry as described in the method. (a) The $\%$ cell population of each phase under different treatments, (b) The flow cytogram profiles of the treatments.

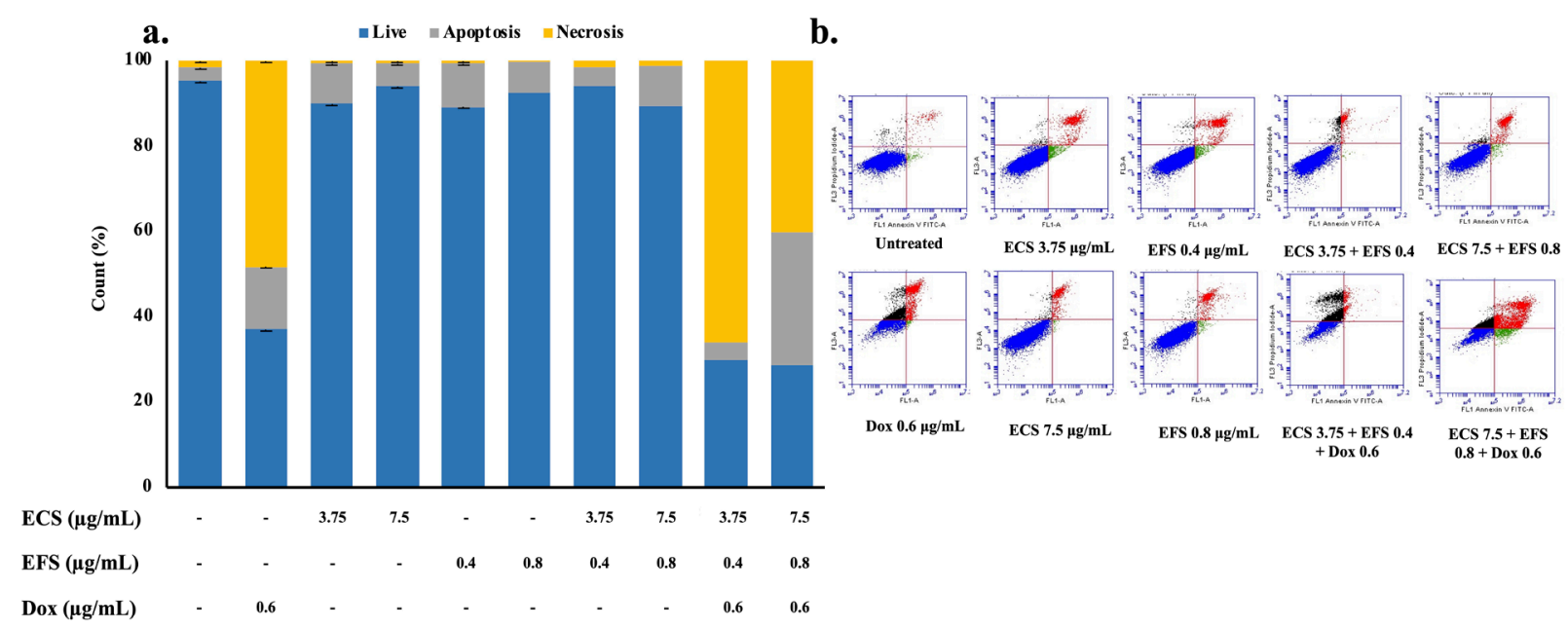

Figure 3. Effect of ECS, EFS, Dox, as well as Their Combinations on Apoptosis of 4T1 Cells. Flow cytometry based assay was performed on $24 \mathrm{~h}$ treated cells as described at the method. (a) The quantification of \% apoptosis cells (b) the flow cytograms of each treatment. The results were displayed as average \pm standard deviation $(n=3)$.

phosphorylated $\mathrm{I} \kappa \mathrm{B} \alpha$, indicating that all the agents might inhibit the phosphate transfer from ATP to I $\kappa \mathrm{B} \alpha$ catalyzed by IKK. If we looked more detail at the intensities, the combination treatments exhibited stronger effects in decreasing the phosphorylation of $\mathrm{I} \kappa \mathrm{B} \alpha$, suggesting there is a synergistic effect of their combination in inhibiting the activity of IKK.

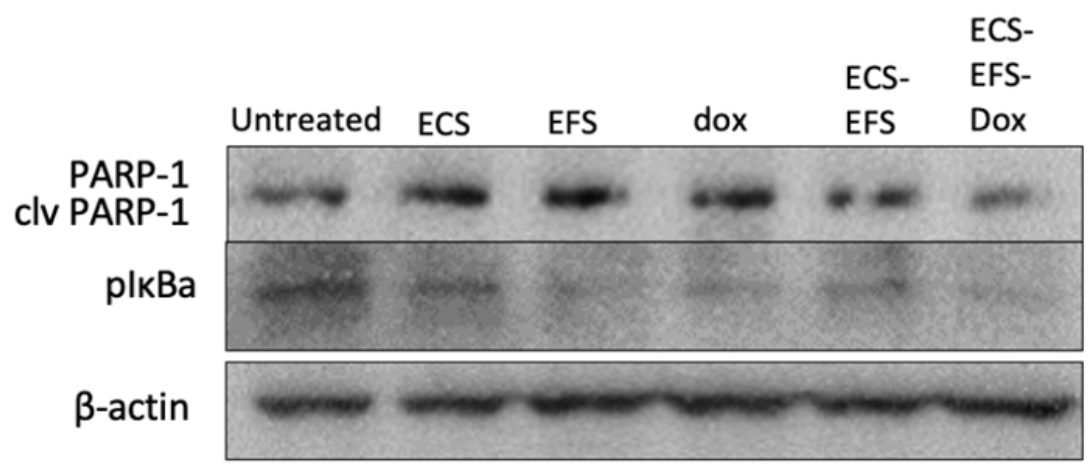

Figure 4. Protein Expression in 4T1 Cells Related to Cell Cycle and Apoptosis. Using Western Blotting of ECS $10 \mu \mathrm{g} / \mathrm{mL}$, EFS $1.7 \mu \mathrm{g} / \mathrm{mL}$, and doxorubicin $0.23 \mu \mathrm{g} / \mathrm{mL}$ treatments, then the expressions of PARP-1 (upper), and $\mathrm{p}$ - I $\mathrm{B} \alpha$ (middle) with control normalized of b-actin (Bottom) were evaluated. 


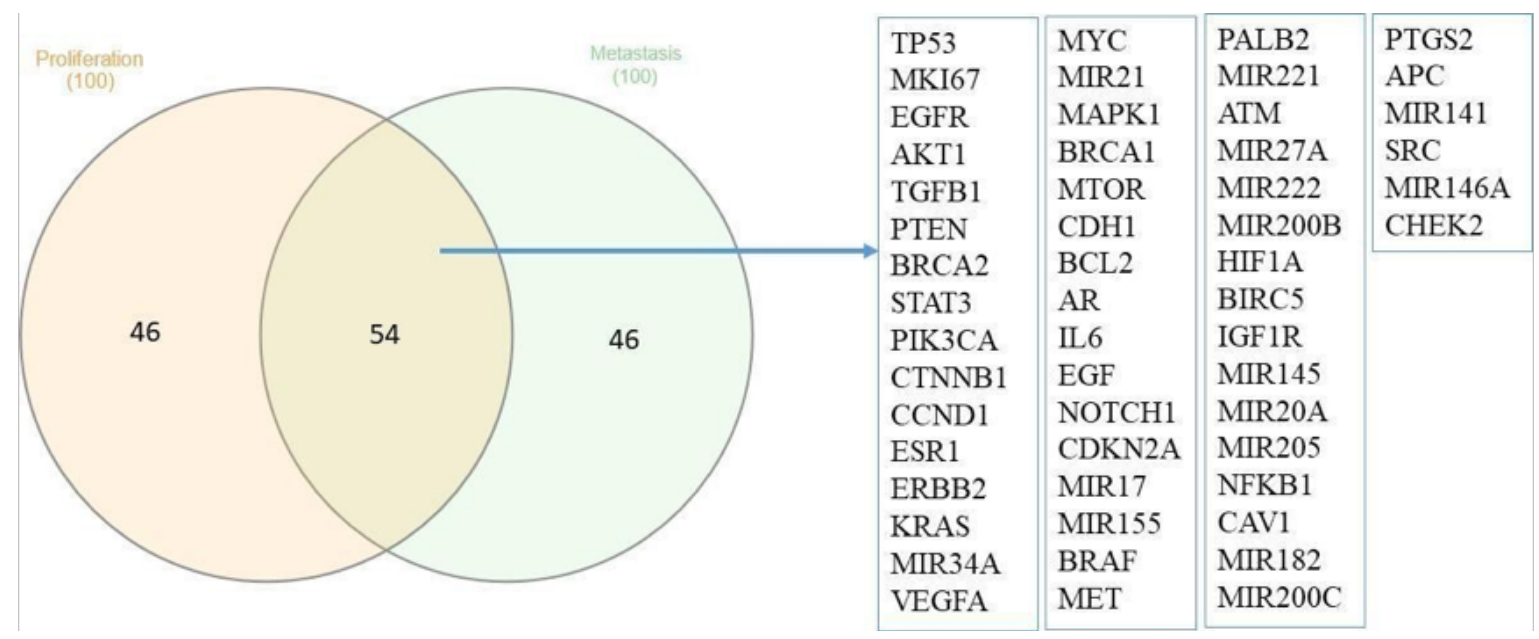

Figure 5. Gene Expression Analysis of Cell Proliferation and Metastasis in TNBC

\section{Bioinformatics Study}

To identify the most influential genes or proteins in driving cell proliferation and metastasis in TNBC, we did data mining and analyzed through a Genecards database. We found more than 1,000 genes marked in each physiological process. We then analyzed the top 100 of each by using Interactivenn to find out the same genes or proteins involved in both processes. There were 54 genes shared in both cell proliferation and cell metastasis covering membrane receptors of a signal transducer, molecule signaling, and factor transcriptions, including NF- $\kappa \mathrm{B}$ (Figure 5).

\section{Molecular docking simulations}

As we found that NF- $\kappa \mathrm{B}$ might play a role in cell proliferation and metastasis of TNBC cells, we then analyzed the key protein in the activation of NF- $\kappa$ B. IKK is the protein kinase responsible for the phosphorylation of $\mathrm{I} \kappa \mathrm{B} \alpha$ that we obtained was decreased under the combination treatment. Therefore, we analyzed whether or

a.

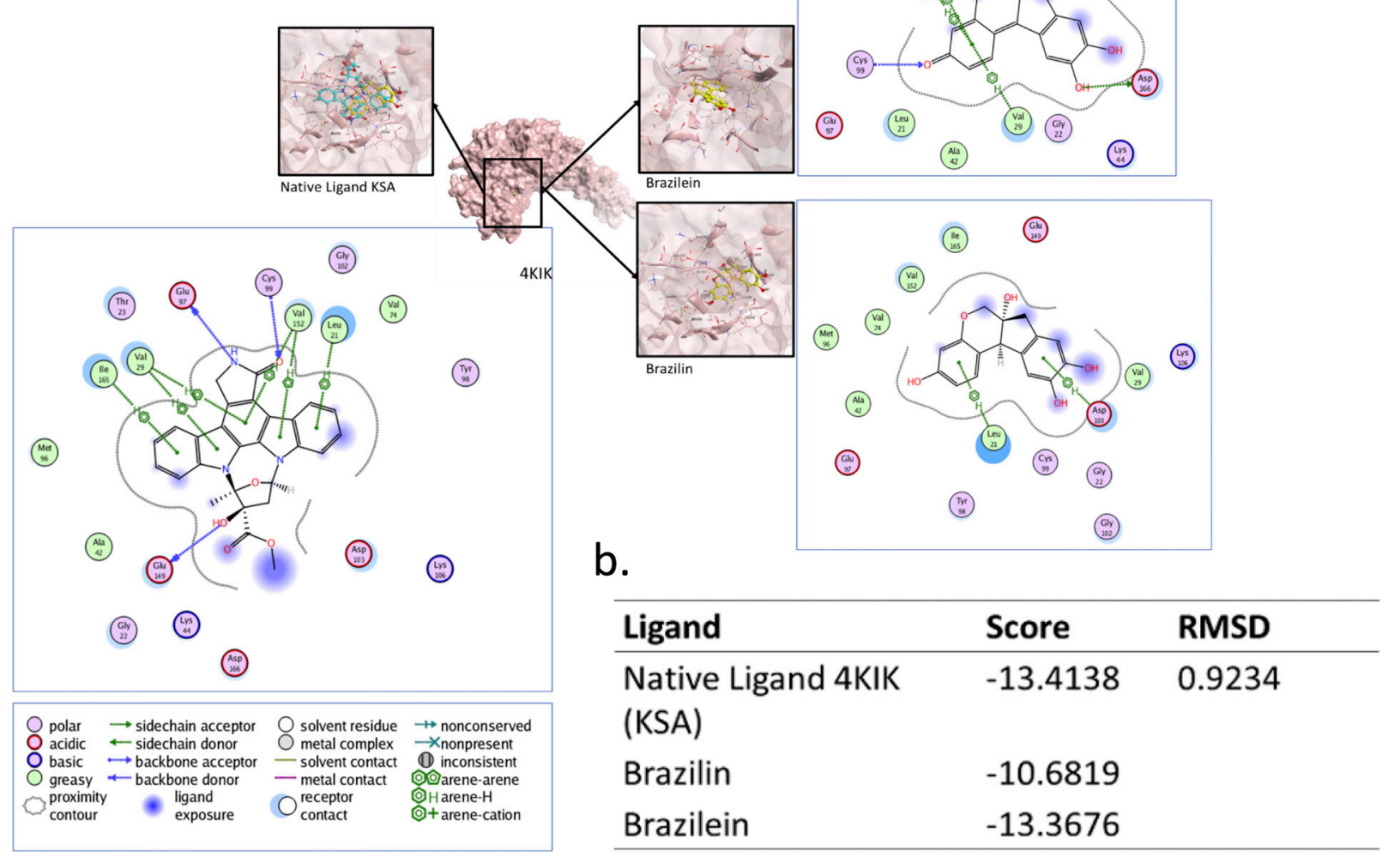

Figure 6. Docking Simulation of Brazilin and Brazilein to IKK Protein. Molecular dockings were performed using MOE software as described in the method. (a) The visualization of an interactive model between the compounds and the IKK protein in the kinase pocket. (b) Table of RMSD and docking scores 
a.

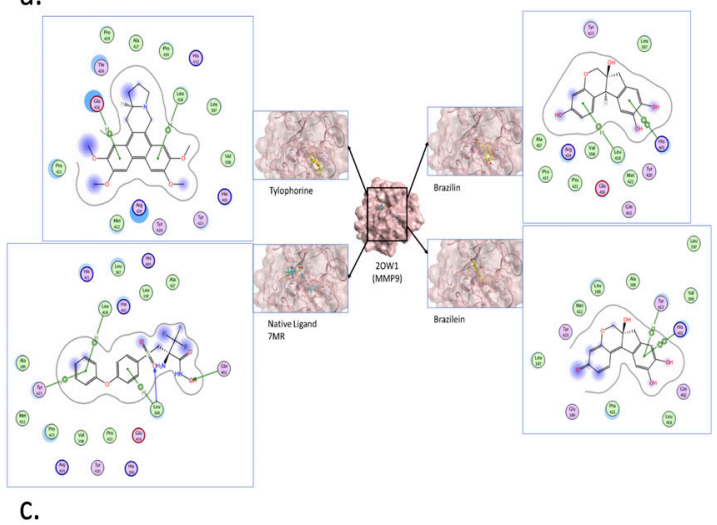

c.

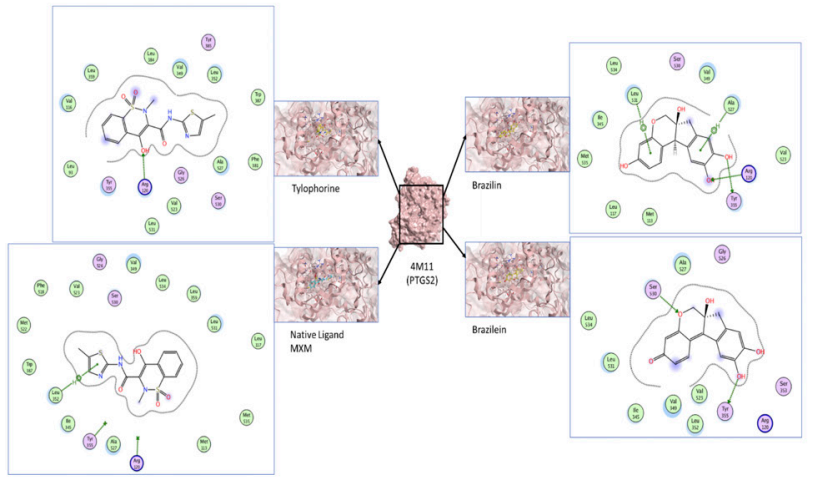

b.

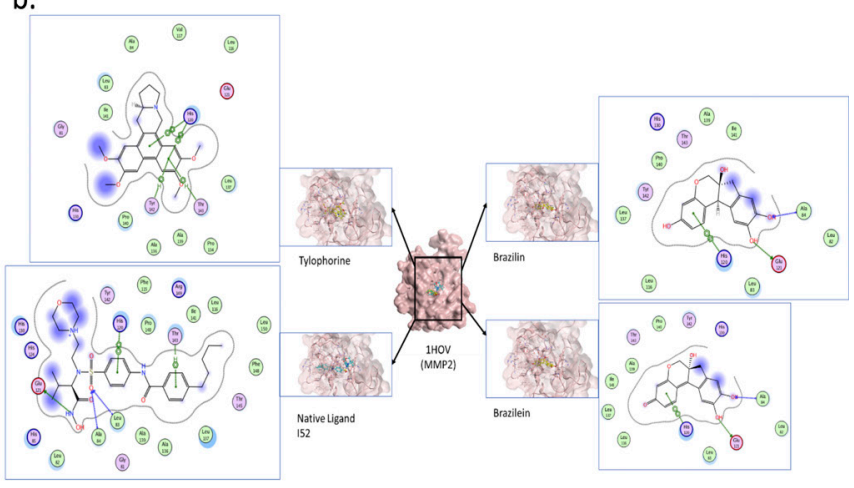

d.

Figure 7. Docking Simulation of Brazilin, Brazilein, and Tylophorine to MMP2 (PDB ID:1HOV), MMP9 (PDB ID:2OW1), and PTGS2 (PDB ID: 4M11) Proteins. Molecular dockings were performe using MOE software as described in the method. (a-c) The visualization of an interactive model between the compounds and the target proteins (MMP9, MMP2, and PTGS2 respectively) at the active sites. (d) Table of RMSD and docking scores.

not the active compounds of C. sappan namely brazilin and brazilein and F. septica namely tylophorine were targeted on IKK protein. Brazilein is the oxidized form of brazilin, and both compounds are the major compounds of $C$. sappan and have significant pharmacological activity. Therefore, we performed molecular docking to characterize the interaction between each compound to IKK protein (PDB ID 4KIK) in the kinase domain and compared them with KSA as the native ligand (Figure 6a). All interactions are

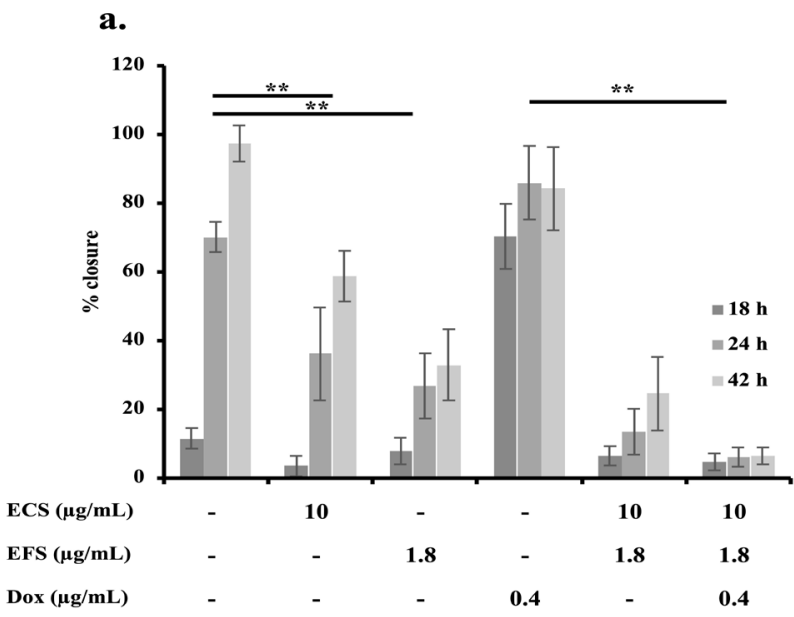

considered to be valid when they have the RMSD values of less than $2 \mathrm{~A}$. Both brazilin and brazilein exhibited the same amino acid residues environment compared to the KSA native ligand, indicating that they had the same bonding pocket that might signify their inhibitory activities. In these cases, brazilein exhibited relatively the same docking score compared to KSA, the standard inhibitor, meanwhile, brazilin performed less (Figure $6 b)$. This finding indicated that brazilein might be a good

b.

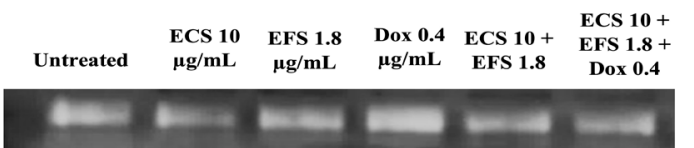

c.

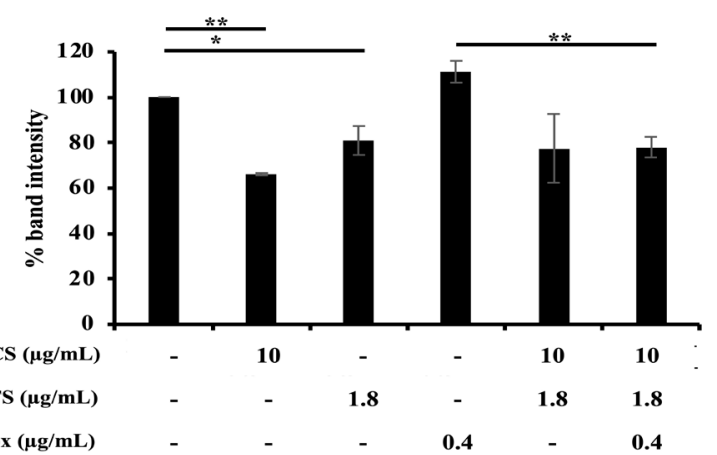

Figure 8. Effects of ECS, EFS, Dox, as well as Their Combinations on Cell Migration of 4T1. (a) Percentage of gap closure (\% closure) single treatment of ECS, EFS, and doxorubicin and their combinations. (b) MMP9 expression levels were determined by gelatin zymography assay. (c) The relative band intensity of MMP9 expression against the untreated cells. 
a.

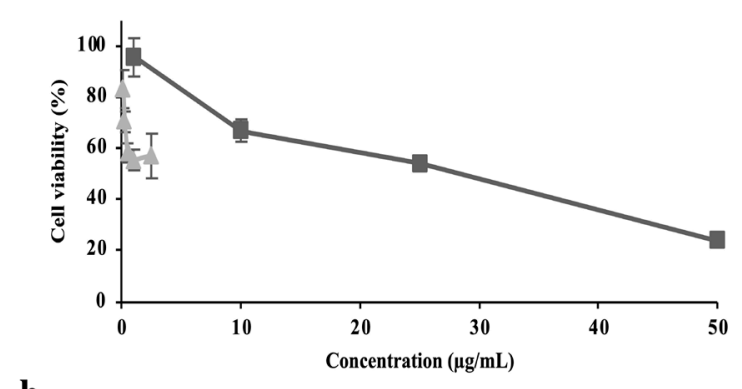

b.

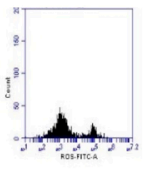

Untreated

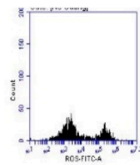

Dox $0.6 \mu \mathrm{g} / \mathrm{mL}$

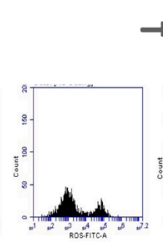

ECS $3.75 \mu \mathrm{g} / \mathrm{mL}$

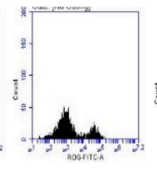

$\operatorname{ECS} 7.5 \mu \mathrm{g} / \mathrm{mL}$
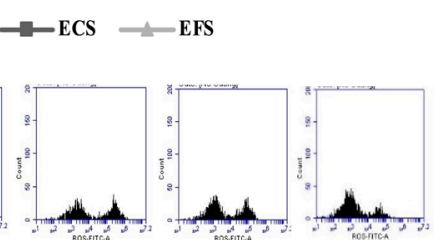

EFS $0.4 \mu \mathrm{g} / \mathrm{mL} \quad$ ECS $3.75+$ EFS 0.4 ECS $7.5+$ EFS 0.8
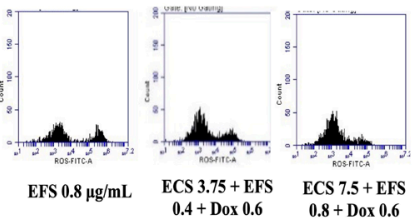

c.

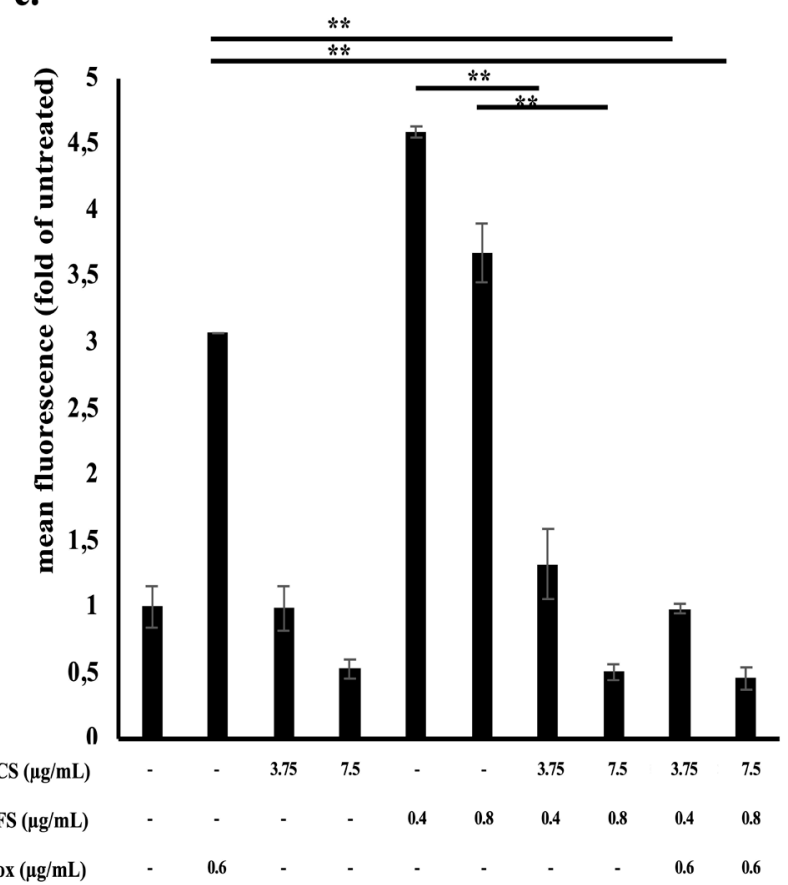

Figure 9. Effects of ECS, EFS, Dox, as well as Their Combinations on ROS Generation in Vero Cells. (a) Growth inhibitory profiles of ECS and EFS treatments. The $\mathrm{IC}_{50}$ values ECS and EFS are $29 \mu \mathrm{g} / \mathrm{mL}$, and $3 \mu \mathrm{g} / \mathrm{mL}$ respectively. (b) the flow cytograms of each treatment. (c) The level of ROS intracellular of Vero cells following ECS, EFS, and Dox treatment. The mean fluorescence is used to represent the results $\left({ }^{*} \mathrm{p}<0.01,{ }^{*} \mathrm{p}<0.001\right)$.

inhibitor for IKK to attenuate the activation of NF- $\mathrm{NB}$.

$\mathrm{NF}-\kappa \mathrm{B}$ is known as the major transcription factor for MMP-9, MMP-2, and PTGS2 that plays important roles in cell migration and metastasis (Arkan and Greten, 2011). Therefore, we then examined the possibility of brazilin, brazilein, and tylophorine in inhibiting those enzymes through binding to their active sites. We demonstrated that those compounds bound strongly to the protein targets at the ligand-binding sites using molecular docking. Almost all the compounds performed better bonding compared to the native ligands. These findings indicated that the three compounds might inhibit the enzyme activities through the binding position at the active site of the enzymes. In these cases, brazilin and brazilein showed better binding activities compared to tylophorine (Figure 7).

Effects of ECS, EFS, as well as their combinations on cell migration

The anti-metastatic and anti-migratory effects of ECS and EFS were then investigated using the same 4T1 cell line, as it is metastatic and has a strong migratory characteristic. The combination of ECS and EFS treatment prevented the occurrence of closure with a low percentage. This evidence showed that both ECS and EFS were capable of inhibiting 4T1 cell migration. Meanwhile, doxorubicin caused migration with a high closure percentage, even at $18 \mathrm{~h}$ (Figure 8a). This result is consistent with the evidence that has been widely reported. When ECS and EFS were combined with doxorubicin, the inhibitory effect of both was significantly stronger. As a result, both can effectively inhibit doxorubicin-induced migration.
Gelatin Zymography was used to confirm whether the two extracts had any effect on MMP-9 expression. MMP-9 expression was reduced by ECS and EFS, whereas doxorubicin showed similar results to the untreated cells. When compared to doxorubicin, the combination of the three samples reduced the MMP-9 expression. As a result, in addition to preventing migration, both extracts can also inhibit the expression of MMP-9 induced by doxorubicin.

Effects of ECS, EFS, as well as their combinations on the cytotoxicity and ROS level of Vero cells

Finally, we examined the effects of the two extracts and their combinations on normal kidney Vero cell line. Single treatment of ECS on Vero cells showed no cytotoxic effects with $\mathrm{IC}_{50}$ value of $29 \mu \mathrm{g} / \mathrm{mL}$ which is considered as not favorable on non-cancer cells. Whereas, EFS gave cytotoxic effects with the $\mathrm{IC}_{50}$ value of $3 \mu \mathrm{g} / \mathrm{mL}$, but showed a selectivity index of more than 2 , indicating that EFS has a high selectivity against cancer cells. DCFDA staining assay showed that only ECS $7.5 \mu \mathrm{g} / \mathrm{mL}$ was able to reduce intracellular ROS level in Vero cells. Meanwhile, EFS with both concentrations significantly increased ROS level. On the contrary, combination treatment of both extracts with doxorubicin significantly reduced intracellular ROS level induced by doxorubicin. These results indicated that both extracts exhibit ROS modulation effects in Vero cells that will be beneficial as nephro-protection.

\section{Discussion}


This study explored the cytotoxic effects of combination treatment of ECS and EFS against the proliferating 4T1 cells. We found that single treatment of ECS and EFS performed a strong cytotoxic effect and their combination in relatively low doses provided a significant enhancement effect $(p<0.01)$. Moreover, when they were combined with doxorubicin, the growth suppression effect on 4T1 cells was increased significantly $(\mathrm{p}<0.01)$. These findings indicated that this combination treatment performs a synergistic effect that may be contributed by their active compounds targeting the physiological process of the cancer cells. However, they did not seem to be affecting the cell cycle progression as well as inducing apoptosis, except in the small portion of the combination treatment with doxorubicin. This may happen due to the experiment not using synchronized cell lines. The other possible reason is that the apoptosis evidence might have occurred before $24 \mathrm{~h}$ observation that it was not examined in this experiment. Nevertheless, we could predict that the existing compounds of the extracts may target several proteins involved in all phases of the cell cycle progression that would be interesting to be explored further.

The enhancement effects in combination treatments of the two extracts with doxorubicin are also interesting for their prospect to be used as co-chemotherapeutic agents. In this perspective, we hope that both herbs would be applicable due to their safety properties as common herbs that have been used for daily consumption and medicinal herbs. In the molecular mechanism view, we also noted that the chemical constituents of C. sappan and F. septica gave a contribution to the target mechanisms in inhibiting cell proliferation and metastasis. Molecular docking simulation to IKK protein confirmed that brazilein exhibited high affinity compared to the standard inhibitor. IKK is the upstream line of the NF- $\kappa \mathrm{B}$ activation that plays a role in triggering many gene expressions involved in several physiological tumorigenesis (Arkan and Greten, 2011), such as cell proliferation and metastasis (Mussbacher et al., 2019). The analysis of gene markers of cell proliferation and metastasis support this hypothesis, relaying several genes that are connected to the $\mathrm{NF}-\kappa \mathrm{B}$ activation such as ERBB2, EGFR, AP1, CycD, MMPs, PTGS2, BCl2, and EGF (Shostak and Chariot, 2015). Inhibition of NF- $\kappa \mathrm{B}$ activation by brazilein leads to an attenuation of the expressions of those genes resulting in the inhibition of cell proliferation in several phases and also disrupting the cell migration. In this concern, we confirmed that those combination treatments also inhibited cell migration and MMP-9 expression. In addition, using molecular docking simulation, we found that brazilin and brazilein was bound tightly and better than that of the native ligands to MMP-9, MMP-2, and PTGS2, the three enzymes that play roles in cell migration and metastasis (Ercolano et al., 2019; Webb et al., 2017). This evidence gives insight for further exploration to confirm the inhibiting properties of those compounds to enzyme activities of MMP-9, MMP-2, and PTGS2. Moreover, doxorubicin is known to activate NF- $\kappa B$ triggering cardiotoxicity and cancer cell migration prior to metastasis (Dalmases et al., 2013; Xu et al., 2020) so that this finding could be continued to be explored on the suppressing effect of $C$. sappan against the unexpected effect of doxorubicin.

The activation of NF- $\kappa \mathrm{B}$ by doxorubicin is also known to correlate with intracellular ROS generation that may be dangerous as the cause of cell damage (Guo et al., 2013). Our data confirmed that Vero cells increased ROS levels significantly after treatment with doxorubicin. The Vero cell line is a fibroblast cell type of an African green monkey's kidney. Therefore, this doxorubicin elevating ROS of Vero cells can be a model of kidney damage by oxidative stress due to chemical damaging agents, such as chemotherapeutic agents. Interestingly, the combination treatment of ECS and EFS with doxorubicin reduced intracellular ROS level significantly which might also be correlated with the inhibitory activities of their active compounds against NF- $\kappa \mathrm{B}$ activation through IKK binding as well as their antioxidant properties. Moreover, the two agents performed low cytotoxic activity against the Vero cell line with a selectivity index of more than 2 , indicating that they were selective for cancer cells. These findings figure out the potential development of those herbs as cochemotherapeutic agents to protect kidney damage caused by chemotherapeutic induced-oxidative stress.

Taken together, this study provides evidences that C. sappan, and F. septica are promising to be developed as co-chemotherapeutics agents. Moreover, their main compounds, brazilin, brazilein, and tylophorine, could be the essential markers of the extracts for the application as anti-cancer agents. On the other hand, those compounds can be developed individually or in combination with other agents that synergistically abrogate cell proliferation and metastasis. However, this study is limited to in vitro and virtual approaches and needs further study to reach closely in clinical applications. Nevertheless, we perceive that $C$. sappan and F. septica are relatively safe and have been used for several pharmacological purposes, so the development of new applications, especially for health purposes is challenging.

In conclusion, ECS and EFS displayed cytotoxic properties on 4T1 cells and significantly strengthened the cytotoxic effect of doxorubicin and inhibited cell migration. The increased cytotoxic effect and the inhibition of cell migration of their co-treatment may correlate to the modulation of NF- $\kappa \mathrm{B}$ activation and the inhibition of MMP-9, MMP-2, and PTGS2 activities by the active compounds namely brazilin, brazilein, and tylophorine Moreover, ECS and EFS reduced intracellular ROS level in Vero cells with lower cytotoxicity. Therefore, C. sappan wood and F. septica leaves extracts could be developed as co-chemotherapeutic and nephroprotective agents.

\section{Abbreviations}

ECS: Caesalpinia sappan extract

EFS: Ficus septica extract

Dox: doxorubicin

TNBC: triple-negative breast cancer

MMP: matrix metalloproteinase

ROS: reactive oxygen species

DCFDA: 2',7'-Dichlorodihydrofluorescein diacetate

NF-кB: nuclear factor kappa-light-chain-enhancer of activated $B$ cells

Asian Pacific Journal of Cancer Prevention, Vol 23 
PARP-1: poly [ADP-ribose] polymerase 1

PTGS2: prostaglandin-endoperoxide synthase 2

\section{Author Contribution Statement}

SH performed almost all laboratory experiments and prepared the manuscript, IAS curated bioinformatics analysis and molecular docking, UMZ conducted ROS flow cytometry and data analysis as well as preparing the manuscript, FW supported flow cytometry and data analysis, EM organized the project and idea, validated all the data, and finishing the manuscript.

\section{Acknowledgments}

We thank the Directorate of Research and the Post-Doc program of Universitas Gadjah Mada who provided the funding in finishing this work ((contract No. 6114/UN1. III/DIT.LIT/PT/2021)). Some of data are part of the Dissertation of Sari Haryanti, 2018.

\section{Conflict of interest}

We declare that we do not have a conflict of interest.

\section{References}

André, F, Zielinski, CC (2012). Optimal strategies for the treatment of metastatic triple-negative breast cancer with currently approved agents. Ann Oncol, 23, vi46-51.

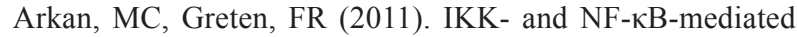
functions in carcinogenesis. Curr Top Microbiol Immunol, 349, 159-69.

Cragg, GM, Pezzuto, JM (2016). Natural products as a vital source for the discovery of cancer chemotherapeutic and chemopreventive agents. Med Princ Pract, 25, 41-59.

Dalmases, A, González, I, Menendez, S, et al (2013). Deficiency in $\mathrm{p} 53$ is required for doxorubicin induced transcriptional activation of NF- $\mathrm{kB}$ target genes in human breast cancer. Oncotarget, 5, 196-210.

Ercolano G, De Cicco P, Rubino V, et al (2019). Knockdown of PTGS2 by CRISPR/CAS9 system designates a new potential gene target for melanoma treatment. Front Pharmacol, 10, 1456.

Finn NA, Kemp ML (2012). Pro-oxidant and antioxidant effects of N-acetylcysteine regulate doxorubicin-induced NF-kappa B activity in leukemic cells. Mol Biosyst, 8, 650-62.

Guo, R-M, Xu W-M, Lin J-C,et al (2013). Activation of the p38 MAPK/NF- $k B$ pathway contributes to doxorubicin-induced inflammation and cytotoxicity in $\mathrm{H} 9 \mathrm{c} 2$ cardiac cells. $\mathrm{Mol}$ Med, 8, 603-8.

Handayani S, Susidarti RA, Jenie RI, Meiyanto E (2017). Two Active Compounds from Caesalpinia sappan L. in Combination with Cisplatin Synergistically Induce Apoptosis and Cell Cycle Arrest on WiDr Cells. Adv Pharm Bull, 7, 375-80.

Haryanti, S, Pramono, S, Murwanti, R, Meiyanto, E (2016). The synergistic effect of doxorubicin and ethanolic extracts of Caesalpinia sappan L. wood and Ficus septica Burm. f. leaves on viability, cell cycle progression, and apoptosis induction of MCF7 cells. Indones J Biotechnol, 21, 29-37.

Hidayati, DN, Jenie, RI, Meiyanto, E (2018). Combination of Curcuma (Curcuma xanthorriza Roxb) rhizome ethanolic extract and ewar-ewar (ficus septica Burm.F) leaves ethanolic extract increases cisplatin cytotoxicity on T47D breast cancer cells through cell cycle modulation. Indones J Cancer Chemoprevention, 8, 120-5.

Hsieh C-Y, Tsai P-C, Chu C-L, et al (2013). Brazilein suppresses migration and invasion of MDA-MB-231 breast cancer cells. Chem Biol, 204, 105-15.

Jenie RI, Handayani S, Susidarti RA, Udin LZ, Meiyanto E (2018). The cytotoxic and antimigratory activity of BrazilinDoxorubicin on MCF-7/HER2 cells. Adv Pharm Bull, 8, 507-16.

Landis-Piwowar KR, Iyer NR (2014). Cancer chemoprevention: Current State of the Art. Cancer Growth Metastasis, 7, $19-25$.

Ma J, Wang Y, Zheng D, et al (2013). Rac1 signalling mediates doxorubicin-induced cardiotoxicity through both reactive oxygen species-dependent and -independent pathways. Cardiovasc Res, 97, 77-87.

Meiyanto E, Putri H, Arum Larasati Y, et al (2019). Anti-proliferative and Anti-metastatic Potential of Curcumin Analogue, Pentagamavunon-1 (PGV-1), Toward Highly Metastatic Breast Cancer Cells in Correlation with ROS Generation. Adv Pharm Bull, 9, 445-52.

Mussbacher M, Salzmann M, Brostjan C, et al (2019). Cell Type-Specific Roles of NF- $\mathrm{B}$ B Linking Inflammation and Thrombosis. Front Immunol, 10, 85.

Nugroho AE, Hermawan A, Nastiti K, et al (2012). Immunomodulatory effects of hexane insoluble fraction of Ficus septica Burm. F. in doxorubicin-treated rats. Asian Pac J Cancer Prev, 13, 5785-90.

Qin J, Wang Y, Bai Y, et al (2012). Epigallocatechin-3-gallate inhibits bladder cancer cell invasion via suppression of NF- $\kappa \mathrm{B}-$ mediated matrix metalloproteinase- 9 expression. Mol Med Rep, 6, 1040-4.

Rizeq B, Gupta I, Ilesanmi J, et al (2020). The power of phytochemicals combination in cancer chemoprevention. J Cancer, 11, 4521-33.

Santoso RA, Lienaningrum AS, Bangun ED, et al (2019). Black cumin (Nigella sativa L.) and awar-awar (Ficus septica burm. F.) combination extract inhibits proliferation and modulates cell cycle on HeLa cell. AIP Conf Proc, 2099, 020023.

Shostak K, Chariot A (2015). EGFR and NF-kB: partners in cancer. Trends Mol Med, 21, 385-93.

Wahba HA, El-Hadaad HA (2015). Current approaches in treatment of triple-negative breast cancer. Cancer Biol Med, 12, 106-16.

Wang W, Nag SA, Zhang R (2015). Targeting the NFאB signaling pathways for breast cancer prevention and therapy. Curr Med Chem, 22, 264-89.

Webb AH, Gao BT, Goldsmith ZK, et al (2017). Inhibition of MMP-2 and MMP-9 decreases cellular migration, and angiogenesis in in vitro models of retinoblastoma. $B M C$ Cancer, 17, 434.

Xu A, Deng F, Chen Y,et al (2020). NF-kB pathway activation during endothelial-to-mesenchymal transition in a rat model of doxorubicin-induced cardiotoxicity. Biomed Pharmacother, 130, 110525.

Yin L, Duan J-J, Bian X-W, Yu S-C (2020). Triple-negative breast cancer molecular subtyping and treatment progress. Breast Cancer Res, 22, 61.

Zhao Y, Yang F, Li W, et al (2017). miR-29a suppresses MCF7 cell growth by downregulating tumor necrosis factor receptor 1. Tumour Biol, 39, 1010428317692264.

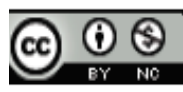

This work is licensed under a Creative Commons AttributionNon Commercial 4.0 International License. 